\title{
A mimic of phosphorylated prolactin induces apoptosis by activating AP-1 and upregulating p21/waf1 in human prostate cancer $\mathrm{PC} 3$ cells
}

\author{
LIANLIAN DU ${ }^{1}$ and WEI WU $\mathrm{W}^{1,2}$ \\ ${ }^{1}$ Department of Epidemiology and Health Statistics, School of Public Health and Family Medicine; \\ ${ }^{2}$ Department of Biochemistry and Molecular Biology, School of Basic Medical Sciences, \\ Capital Medical University, Beijing 100069, P.R. China
}

Received March 28, 2012; Accepted July 9, 2012

DOI: $10.3892 / \mathrm{ol} .2012 .876$

\begin{abstract}
A mimic of phosphorylated prolactin (S179D PRL) has been demonstrated to inhibit prostate cancer cell growth in vitro and in vivo; however, the mechanisms involved in this process remain unknown. In this study, we identified that a four-day treatment of S179D PRL $(1 \mu \mathrm{g} / \mathrm{ml})$ in human prostate PC3 cancer cells activated JNK, c-fos and c-jun, and led to apoptosis. We also demonstrated that $\mathrm{p} 21 /$ waf1 was upregulated in cells transfected with the human PRL receptor (S1b) following a four-day incubation with S179D PRL $(1 \mu \mathrm{g} / \mathrm{ml})$. Once the cells were cotransfected with S1b and either c-fos, c-jun or the c-fos/c-jun constructs for $24 \mathrm{~h}$, S17D PRL activated $\mathrm{JNK}, \mathrm{c}$-fos and c-jun, and induced apoptosis in the c-fos/c-jun transfected cells. Additionally, S179D PRL upregulated p21 luciferase activity in the cells transfected with the S1b, activating protein-1 (AP-1) (7x) Luc or p21 Luc constructs. SP600125 $(25 \mu \mathrm{M})$, a JNK blocker, inhibited the upregulation of AP-1 Luc and p21 Luc in the c-fos/c-jun transfected cells. These results demonstrate that S179D PRL activates JNK and AP-1, which leads to 21 upregulation and apoptosis in human prostate PC3 cancer cells.
\end{abstract}

\section{Introduction}

Overexpression of unmodified prolactin (U-PRL) promotes prostate and breast cancer; however, phosphorylated PRL has the opposite effect (1). A recombinant PRL was constructed and used as a mimic of the phosphorylated PRL (S179D PRL). In a previous study, we demonstrated that S179D PRL

Correspondence to: Dr Wei Wu, Department of Biochemistry and Molecular Biology, School of Basic Medical Sciences, Capital Medical University, 10 Youanmenwai Avenue, Beijing 100069, P.R. China

E-mail: weiwu207@ccmu.edu.cn

Key words: prolactin, MAPK, p21, apoptosis inhibited breast and prostate cancer cell growth (2). The mechanisms involved were associated with short PRL signaling; however, it was suggested that the short PRL receptor (PRLR) did not induce mammary cell differentiation in response to U-PRL. It has been suggested that the short PRLR lacks the structural Stat5 activation site. Nevertheless, a short mouse PRLR was able to activate mitogen-activated protein kinases (MAPKs), which led to the proliferation of NIH/3T3 cells (3). Binart et al revealed that a short form of mouse PRLR rescues mammopoiesis in heterozygous PRLR mice (4). Notably, we demonstrated that S179D PRL induced $\beta$-casein gene expression by regulating the ratio of short to long PRLR in mouse mammary HC11 cells (5). Similarly, Meng et al and Qazi et al identified that a lower ratio of short to long PRLR contributed to breast cancer development, as the short PRLR form inhibited signaling of the long PRLR form via heterodimerization $(6,7)$. Both the long and the short PRLR forms have been identified in human prostate tissues (8). We previously demonstrated that S179D PRL inhibited prostate cancer DU145 cell growth via the short human PRLR, S1b (2), and inhibited the growth of DU145-derived tumor cells in nude mice (9).

In previous studies, we also revealed that S179D PRL activated short PRLR and ERK1/2 resulting in p21/waf1 expression in prostate cancer cells $(2,9)$. It is well known that $\mathrm{p} 21 /$ waf1 induces cell cycle arrest, differentiation and apoptosis in a number of cell lines. These results indicate that S179D PRL may induce apoptosis via the short PRLR S1b and the upregulation of $\mathrm{p} 21 /$ waf1. Short PRLR signaling may trigger the activation of JNK, c-jun and c-fos, resulting in the activation of activating protein-1 (AP-1) and the upregulation of p21/waf1 in human prostate cancer cells. Previous studies have demonstrated that the accumulation of ERK1/2 resulted in the upregulation of p21 (10-12). Structurally, the short PRLR has the potential to activate Jak 2 and further activate MAPKs. Studies have revealed that the short PRLR activates AP-1 family members, including c-jun and c-fos complexes, and U-PRL induces an increase in the c-jun content of the AP-1 transcriptional complex via activating JNK $(13,14)$. AP-1 was considered to be a regulator of cell death, and p21/waf1 expression correlates with the expression of AP-1 family members 
(c-jun and c-fos) in breast cancer cells (15). Additionally, it has been demonstrated that JNK activation, as well as c-jun and $\mathrm{c}$-fos upregulation, contribute to $\mathrm{p} 21 / \mathrm{waf} 1$ expression in selenite-induced HepG2 cell apoptosis. In the present study, we investigated whether S179D PRL activates JNK, c-jun and c-fos, leading to p21/waf1 upregulation and apoptosis in response to S179D PRL in the PC3 cells transfected with human short PRLR S1b. The JNK blocker, SP600125, was used to determine whether JNK signaling contributes to p21/waf1 upregulation. The aim of this study was to uncover the molecular mechanisms associated with S179D PRL and prostate cancer cells.

\section{Materials and methods}

Recombinant PRL. DNA cloning and mutagenesis techniques were used to produce U-PRL and S179D PRL, as previously described (5). S179D PRL was generated by substituting an aspartate for a normally phosphorylated serine. The proteins were expressed and purified, and their activity was studied in an Nb2 cell bioassay. U-PRL promotes Nb2 cell proliferation, while S179D PRL antagonizes this effect.

Cell lines and constructs. The PC3 cell line was purchased from the American Type Culture Collection (ATCC; Manassas, VA, USA). Cells were maintained in RPMI-1640 medium containing $10 \%$ fetal bovine serum (FBS; Invitrogen, Carlsbad, CA, USA) and antibiotics. The constructs pEF4C-S1b and pEF6-LPRLR containing prolactin receptors were gifts from Dr Barbara K. Vonderhaar (National Institute of Health, National Cancer Institute, Bethesda, MD, USA) and the $\beta$-galactosidase ( $\beta$-gal) plasmid was provided by Dr Linda A. Schuler (University of Wisconsin, Madison, WI, USA). AP-1 (7x) was purchased from Stratagene Corp. (La Jolla, CA, USA). The constructs pRSV-jun and pRSV-fos were gifts from Dr Robert Tjian (University of California, Berkeley, CA, USA). The construct p21 Luc was obtained from Dr Leonard P. Freedman (Memorial Sloan-Kettering Cancer Center, New York, NY, USA).

Cotransfection and luciferase assay. PC3 cells were grown in RPMI-1640 medium (Invitrogen) containing 10\% charcoal-stripped horse serum (Cocalico Biologicals, Reamstown, PA, USA), $100 \mathrm{U} / \mathrm{ml}$ penicillin and $100 \mu \mathrm{g} / \mathrm{ml}$ streptomycin (Invitrogen). Cells were grown in six-well plates and transfection was conducted once the cells reached $50-70 \%$ confluency. The $\beta$-gal plasmid $(0.25 \mu \mathrm{g} / 5 \mathrm{ml})$ was cotransfected in order to examine the transfection efficiency. After cells were treated with PRL (U-PRL and S179D PRL; $1 \mu \mathrm{g} / \mathrm{ml}$ ) for three days, the JNK blocker, SP600125 (25 $\mu \mathrm{M}$; Calbiochem, San Diego, CA, USA), was used to inhibit AP-1 Luc and p21 Luc expression in response to each PRL. In another transfection study involving c-fos and c-jun transfection, c-fos $(0.5 \mu \mathrm{g} / 5 \mathrm{ml})$ only, c-jun $(0.5 \mu \mathrm{g} / 5 \mathrm{ml})$ only and c-fos $(0.25 \mu \mathrm{g} / 5 \mathrm{ml})$ plus c-jun $(0.25 \mu \mathrm{g} / 5 \mathrm{ml})$, AP-1 Luc $(0.25 \mu \mathrm{g} / 5 \mathrm{ml})$ or p21 luciferase construct $(0.25 \mu \mathrm{g} / 5 \mathrm{ml})$, short PRLR $(0.25 \mu \mathrm{g} / 5 \mathrm{ml})$ and $\beta$-gal plasmids were used to transfect the cells. In the RPMI-1640 medium containing 5\% charcoal-stripped horse serum, cells were treated with U-PRL or S179D PRL for $24 \mathrm{~h}$. A volume of $10 \mu \mathrm{l}$ was applied to test luciferase activity using a illuminom- eter, following the Promega Luciferase assay manufacturer's instructions.

Preparation of whole cell and nuclear extracts. Cells were rinsed with phosphate-buffered saline (PBS) and scraped off in a buffer containing $20 \mathrm{mM}$ Tris- $\mathrm{HCl}$ (pH 7.4), $140 \mathrm{mM}$ $\mathrm{NaCl}, 0.05 \mathrm{mM}$ EDTA, $10 \mu \mathrm{g} / \mathrm{ml}$ leupeptin, $10 \mu \mathrm{g} / \mathrm{ml}$ aprotinin, $25 \mu \mathrm{g} / \mathrm{ml}$ pepstatin, $1 \mathrm{mM}$ PMSF, $1 \mathrm{mM} \mathrm{Na} \mathrm{VO}_{4}$, $10 \mathrm{nM}$ NaF, $1 \mathrm{mM}$ EGTA and 1\% NP-40. The cell lysate was homogenized and centrifuged at 12,000 x g for $5 \mathrm{~min}$, and the supernatant was considered as a whole cell extract. The whole cell lysate was used to examine the phosphorylated c-jun (p-c-jun), c-fos (p-c-fos) and JNK (p-JNK). If the cells were scraped into a hypotonic buffer, consisting of $10 \mathrm{mM}$ Tris (pH 7.4), $10 \mathrm{mM} \mathrm{NaCl}, 6 \mathrm{mM} \mathrm{MgCl} \mathrm{m}_{2}, 1 \mathrm{mM}$ dithiothreitol and $0.1 \mathrm{mM} \mathrm{Na}_{3} \mathrm{VO}_{4}$, and disrupted with a Dounce homogenizer, the supernatant was removed following centrifugation at $12,000 \times \mathrm{g}$ for $5 \mathrm{~min}$. The pellet from the centrifugation was resuspended in hypertonic extraction buffer, consisting of $20 \%$ glycerol, $20 \mathrm{mM}$ HEPES (pH 7.9), $420 \mathrm{mM} \mathrm{NaCl}, 1.5 \mathrm{mM}$ $\mathrm{MgCl}_{2}, 0.2 \mathrm{mM}$ EDTA, $0.2 \mathrm{mM}$ phenylmethylsufonyl fluoride, $1 \mathrm{mM}$ dithiothreithol and $0.1 \mathrm{mM} \mathrm{Na}_{3} \mathrm{VO}_{4}$, and incubated on ice for $30 \mathrm{~min}$. The supernatant obtained following centrifugation was a nuclear extract. This was used to examine the p21/waf1 protein concentration, which was calculated using the Bradford method.

Western blot analysis. A total of $20 \mu \mathrm{g}$ of protein was loaded onto a reducing SDS-PAGE gel. Following electrophoresis, the protein was transferred to nitrocellulose membranes in $48 \mathrm{mM}$ Tris, $39 \mathrm{mM}$ glycine, $0.1 \%$ SDS and $20 \%$ methanol ( $\mathrm{pH} 8.3$ ). Membranes were blocked with $5 \%$ non-fat milk in washing buffer consisting of Dulbecco's PBS (DPBS; Invitrogen) and 0.1\% Tween-20. Blotted and blocked membranes were probed with primary rabbit polyclonal anti-p21/waf1 (1:500), anti-c-fos (1:1000), anti-p-c-jun (1:1000; Santa Cruz Biotechnology, Inc., Santa Cruz, CA, USA) and anti-JNK (1:500; Promega, Madison, WI, USA) in washing buffer for $3 \mathrm{~h}$ at room temperature or overnight at $4^{\circ} \mathrm{C}$. After washing three times for $15 \mathrm{~min}$ each, the blots were incubated in goat anti-rabbit conjugated to horseradish peroxidase (Sigma, St. Louis, MO, USA) at a dilution of 1:2000-1:10000, as appropriate, for 30-45 min at room temperature. Following a further three washes, the membranes were treated with ECL reagent (Amersham Biosciences, Piscataway, NJ, USA) and an autoradiograph image was obtained.

Apoptosis assay. The treated PC3 cells were collected to examine the DNA degradation. DNA was isolated using phenol/chloroform extraction followed by ethanol precipitation procedures. A total of $10 \mu \mathrm{g}$ of DNA was run on a $0.8 \%$ agarose gel. Apoptosis was determined by DNA fragmentation.

Statistical analysis. Data were subjected to analysis of variance with post-tests for comparison among specific groups using the INSTAT program (Graph PAD Software, San Diego, CA, USA). Post-tests comparing each potential pair of groups were conducted. Bonferroni corrections for multiple compari- 


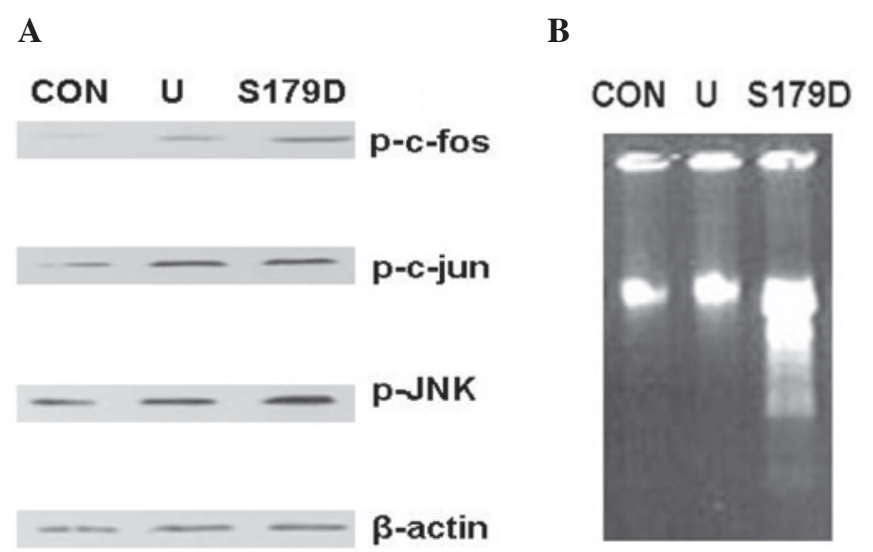

Figure 1. PC3 cells were incubated with U-PRL or S179D PRL for four days. (A) Western blot analysis demonstrated that U-PRL and S179D PRL phosphorylated c-fos, c-jun and JNK. Experiments were conducted in triplicate. $\beta$-actin was used for normalization and equal loading. (B) Genomic DNA was isolated and loaded onto a $0.8 \%$ agarose gel. Smeared-laddered bands indicate the apoptotic effect. S179D PRL triggers apoptosis in PC3 cells; however, U-PRL does not. Experiments were conducted in duplicate. CON, control; U, unmodified; p, phosphorylated; JNK, c-jun N-terminal kinase. PRL, prolactin.

sons against a single group were used. All experiments were conducted a minimum of three times. Following Bonferroni corrections, $\mathrm{P}<0.05$ was considered to indicate a statistically significant difference.

\section{Results}

S179D PRL phosphorylates c-fos, $c$-jun and JNK. PC3 cells were treated with U-PRL or S179D PRL for 4 days. Western blot analysis demonstrated that U-PRL and S179D PRL phosphorylated c-fos, c-jun and JNK (Fig. 1A). Apoptosis assays revealed that S179D PRL induced apoptosis in PC3 cells (Fig. 1B); however, U-PRL did not. Previously, we identified that S179D PRL upregulated the short PRLR S1b and ERK1/2 leading to p21 upregulation in PC3 cells (2). These results indicated that the short PRLR S1b and c-jun/c-fos complex (AP-1) may contribute to S179D PRL signaling and apoptosis.

S179D PRL phosphorylates c-fos, $c$-jun and JNK in the short PRLR S1b transfected cells. It was suggested that S1b activated the MAPK pathway; however, in previous studies, S179D PRL did not upregulate S1b following a $24 \mathrm{~h}$ incubation (2). In this study, we transfected PC3 cells with S1b, and either c-fos, c-jun or c-fos/c-jun constructs, in order to observe the activation of c-fos, c-jun or c-jun/c-fos. Following transfection, cells were treated with U-PRL or S179D PRL. The results demonstrated that U-PRL and S179D PRL activated c-fos, c-jun and JNK in c-fos, c-jun and c-fos/c-jun transfected cells to a certain extent (Fig. 2A). These data reveal that S1b is involved in the activation of c-fos, c-jun and JNK in S179D PRL treated PC3 cells. Additionally, apoptosis was only observed in c-fos/c-jun-transfected cells, but not in the c-fosor c-jun only-transfected cells (Fig. 2B), which suggests that AP-1 contributes to apoptosis.

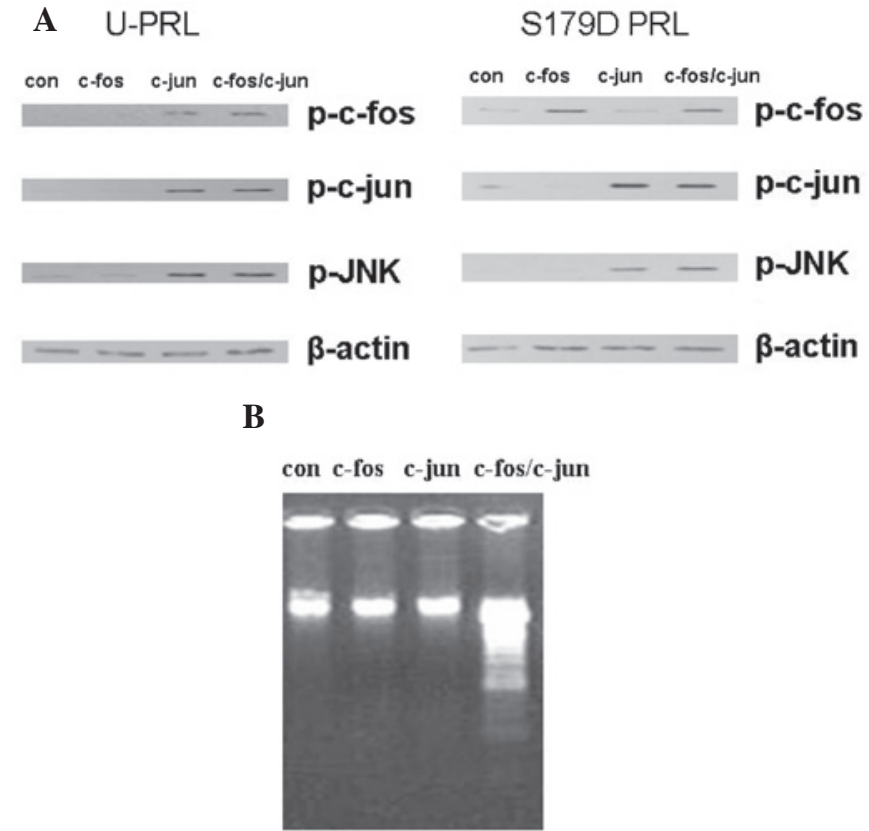

Figure 2. (A) Western blot analysis revealed that U-PRL phosphorylates c-fos, c-jun and JNK after a $24 \mathrm{~h}$ incubation in the c-jun- and c-fos/c-jun-transfected cells but not the c-fos-transfected cells. S179D PRL phosphorylates c-fos, c-jun and JNK after a $24 \mathrm{~h}$ incubation in the c-fos, c-jun and c-fos/c-jun short PRLR S1b-transfected cells. (B) Apoptosis was only observed in the cells transfected with c-fos/c-jun and S1b in response to S179D PRL. All experiments were conducted in duplicate. con, control; U-PRL, unmodified prolactin; p, phosphorylated; JNK, c-jun N-terminal kinase.

S1b triggers p21/wafl upregulation. In this study, we investigated whether S1b contributed to p21/waf1 upregulation. Control PC3 cells were transfected either with or without S1b and cells were treated either with or without S179D PRL. After a 4-day incubation, p21/waf1 was determined using western blot analysis. The results revealed that $\mathrm{p} 21 /$ waf1 was upregulated in S179D PRL-treated cells with S1b transfection (Fig. 3A). We previously identified that S1b was expressed following a 4-day treatment with S179D PRL (2); therefore, S1b contributed to $\mathrm{p} 21 /$ waf1 upregulation in response to S179D PRL.

S179D PRL increases p21 luciferase activity. Activated MAPK (ERK1/2 and JNK) was demonstrated to activate AP-1 complexes (c-jun/c-jun or c-jun/c-fos dimers) in a number of cell types (13-15). In this study, cells were transfected with $\mathrm{S} 1 \mathrm{~b}$, either c-fos, c-jun or c-fos/c-jun and p21 Luc constructs, after 24-h incubation with S179D PRL. The results revealed that S179D PRL increased p21 luciferase activity in c-fos/c-jun transfected cells only (Fig. 3B).

SP600125 inhibits AP-1 or p21 luciferase activity. In this study, we examined the AP-1 (7x) and p21 luciferase activity after PC3 cells were transfected with S1b c-fos/c-jun constructs in response to S179D PRL and the JNK blocker, SP600125 $(25 \mu \mathrm{M})$. After 24-h incubation, the results demonstrated that SP600125 inhibited AP-1 and p21 luciferase activity induced by S179D PRL (Fig. 4A and B). Since the activation of c-fos depends on activated ERK1/2 (15), both 
A
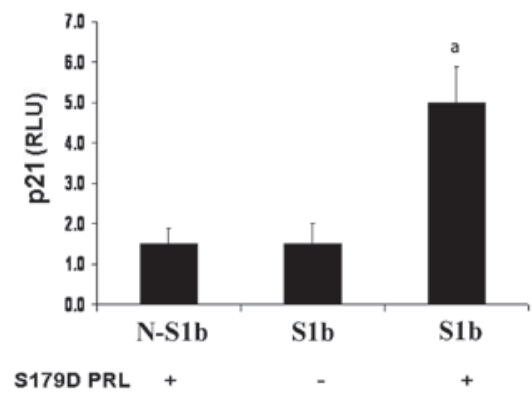

B
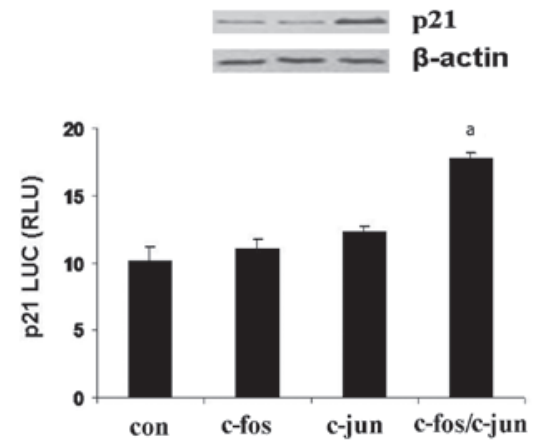

Figure 3. (A) Short PRLR S1b contributed to p21 upregulation. PC3 cells were transfected either with S1b (S1b) or without S1b (N-S1b) for 4 days. Cells were treated with S179D PRL (+) or without S179D PRL (-). After the 4-day incubation, p21 levels were determined by western blot analysis. The data revealed that p21 was upregulated in the S179D PRL-treated cells transfected with S1b, which indicated that short PRLR S1b contributed to $\mathrm{p} 21 /$ waf1 upregulation. $\beta$-actin was used for normalization and equal loading. (B) S179D PRL increases p21 luciferase activity. Cells were transfected with S1b, c-fos, c-jun or fos/c-jun, and the p21 Luc constructs. After a $24 \mathrm{~h}$ incubation with S179D PRL, S179D PRL increased p21 Luc in the c-fos/c-jun-transfected cells. All experiments were conducted in triplicate. ${ }^{a} \mathrm{P}<0.05$ vs. the control cells. RLU, relative units; PRL, prolactin; con, control. PRLR, PRL receptor.

$\mathbf{A}$

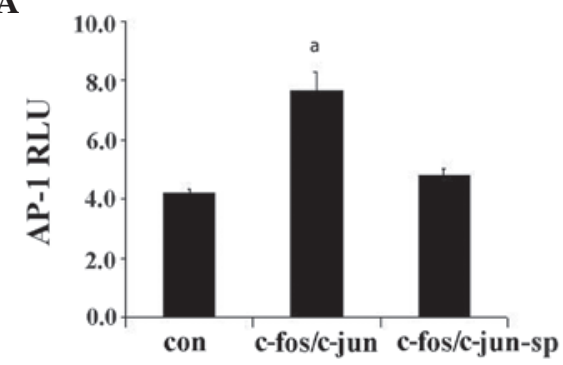

B

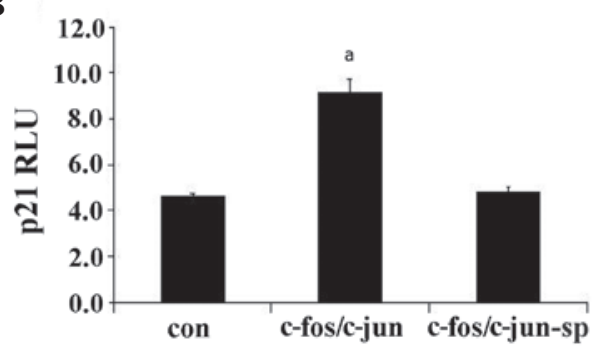

Figure 4. SP600125 inhibits AP-1 and p21 luciferase activities. PC3 cells were cotransfected with S1b, c-fos/c-jun, AP-1 Luc and p21 Luc in response to S179D PRL and/or the JNK blocker, SP600125 (25 $\mu \mathrm{M})$. After a $24 \mathrm{~h}$ incubation, SP600125 inhibited S179D PRL-induced (A) AP-1 and (B) p21/waf1 luciferase activity. This indicates that JNK contributes to the activation of AP-1 and the upregulation of $\mathrm{p} 21$. All experiments were conducted in triplicate. ${ }^{\mathrm{a}} \mathrm{P}<0.05$ vs. the control cells. AP-1, activating protein-1; RLU, relative units; con, control, sp, SP600125. PRL, prolactin; JNK, c-jun N-terminal kinase. activated ERK1/2 and JNK contributed to the activation of AP-1 and p21 upregulation.

\section{Discussion}

In the previous studies, we demonstrated that S179D PRL inhibited prostate cancer in vitro and in vivo $(1,9)$. In the present study, we identified that S179D PRL induced apoptosis via activating AP-1 and upregulating p21/waf1 in PC3 cells. Increased p21 and apoptosis requires short human PRLR S1b and c-fos/c-jun heterodimerization. We previously demonstrated that S179D PRL primarily used the ERK1/2 pathways to activate downstream genes, including Stat5 and p21/waf1 (5). The short PRLR may play a role in signaling. Structurally, the short PRLR has the potential to activate Jak2, which is able to further activate the ERK1/2 pathway. In certain cell types, sustained ERK1/2 activation also leads to p21/waf1 upregulation, cell cycle arrest, differentiation and apoptosis (10-12). In the p21 gene promoter region, there are 2 Stat5 activating sites; however, it is unclear whether S179D PRL upregulates p21/waf1 via Stat5 activation. Serine phosphorylation of Stat5 was revealed to have a more stable binding affinity to the gene promoter (5); therefore, the short PRLR S1b may contribute to Stat5 activation leading to p21/waf1 upregulation and apoptosis. Thus, the short PRLR has the potential to trigger various signaling pathways. Additionally, S179D PRL may induce mRNA alternative splicing to maintain a stable ratio of long and short PRLR (6,7). Studies have demonstrated that activated AP-1 causes a p21/waf1 increase (16).

In the present study, we first identified that S179D PRL upregulated p21, which resulted from c-fos/c-jun heterodimerization. In the c-fos or c-jun only-transfected PC3 cells, p21/waf1 was not upregulated, suggesting that S179D PRL only induced c-fos/c-jun heterodimerization and not c-fos or c-jun homodimerization. The JNK blocker, SP600125, inhibited S179D PRL-induced AP-1 activation and p21/waf1 upregulation by blocking the c-fos/c-jun dimerization. We found that S179D PRL also phosphorylated JNK. Whether the short PRLR is necessary for this activation requires further investigation; however, p21/waf1 upregulation requires the short PRLR S1b. In a previous study, we revealed that the p21/waf1 increase was induced by upregulating the short PRLR S1b and activating ERK1/2. S179D PRL may activate p21/waf1 via activating ERK1/2 and JNK. It is unknown whether MAPK translocates into the nucleus; however, activated c-fos/c-jun (AP-1) was found to induce apoptosis in numerous cell lines, and a particular conformation of the c-fos/c-jun complex is necessary for AP-1 to bind to p21/waf1. Consequently, S179D PRL may inhibit prostate cancer via apoptosis. In these studies, U-PRL also phosphorylated JNK and c-jun; however, no apoptotic effect was identified. JNK is a c-jun N-terminal kinase; therefore, activated JNK may not activate c-fos since c-fos was activated by ERK1/2. U-PRL triggers the Jak2-Stat5 pathway via activating the long PRLR rather than the short PRLR. This effect has been revealed to contribute to cancer cell proliferation $(13,17)$. The short PRLR resulted from the translation of alternatively spliced mRNA. These PRLRs may constitute a regulatory system to regulate the long PRLR signaling. Additionally, S179D PRL, 
a U-PRLR antagonist, may also block the U-PRL signals by competing with the long PRLR. Nevertheless, S179D PRL primarily uses the short PRLR to activate ERK1/2, JNK and transcription factors, leading to p21/waf1 upregulation and apoptosis.

In conclusion, S179S PRL activated c-fos, c-jun and JNK leading to p21/waf1 upregulation and apoptosis, suggesting that S179D PRL may be used as a potential drug to repress prostate cancer.

\section{Acknowledgements}

We are grateful to Dr Ameae Walker, (University of California, Riverside, CA, USA) for her support in the experiments. This study was supported by The Science and Development Plan of Beijing Education Committee (Grant No. KM200910025008) and The National Natural Science Foundation of China (Grant No. 81041067). We would also like to thank Dr Barbara K. Vonderhaar (NCI NIH, Bethesda, MD, USA), Dr Linda A. Schuler (University of Wisconsin, Madison, WI, USA), Dr Leopard Freeman (Memorial Sloan-Kettering Cancer Center, New York, NY, USA) and Dr Robert Tijan (University of California, Berkeley, CA, USA) for their generosity in providing constructs.

\section{References}

1. Wu W, Ginsburg E, Vonderhaar BK and Walker AM: S179D prolactin increases vitamin D receptor and p21 through up-regulation of short $1 \mathrm{~b}$ prolactin receptor in human prostate cancer cells. Cancer Res 65: 7509-7515, 2005.

2. Das R and Vonderhaar BK: Transduction of prolactins (PRL) growth signal through both long and short forms of the PRL receptor. Mol Endocrinol 9: 1750-1759, 1995.

3. Binart N, Imbert-Bollore P, Baran N, Viglietta C and Kelly PA: A short form of the prolactin (PRL) receptor is able to rescue mammopoiesis in heterozygous PRL receptor mice. Mol Endocrinol 17: 1066-1074, 2003.

4. Wu W, Coss D, Lorenson MY, Kuo CB, Xu XL and Walker AM: Different biological effects of unmodified prolactin and a molecular mimic of phosphorylated prolactin involve different signaling pathways. Biochemistry 42: 7561-7570, 2003.

5. Meng JP, Tsai-Morris CH and Dufau ML: Human prolactin receptor variants in breast cancer: Low ratio of short forms to the long-form human prolactin receptor associated with mammary carcinoma. Cancer Res 64: 5677-5682, 2004.
6. Qazi AM, Tsai-Morris CH and Dufau ML: Ligand-independent homo- and heterodimerization of human prolactin receptor variants: inhibitory action of the short forms by heterodimerization. Mol Endocrinol 20: 1912-1923, 2006.

7. Nevalainen MT, Valve EM, Ingleton PM, Nurmi M, Martikainen PM and Harkonen PL: Prolactin and prolactin receptors are expressed and functioning in human prostate. $\mathrm{J}$ Clin Invest 99: 618-627, 1997.

8. Xu X, Kreye E, Kuo CB and Walker AM: A molecular mimic of phosphorylated prolactin markedly reduced tumor incidence and size when DU145 human prostate cancer cells were grown in nude mice. Cancer Res 61: 6098-6104, 2001.

9. Wu W, Zanello L and Walker AM: S179D prolactin sensitizes human prostate cancer cells such that physiological concentrations of 1,25 dihydroxy vitamin $D 3$ result in growth inhibition and cell death. Prostate 67: 1498-1506, 2007.

10. Chen T and Wong YS: Selenocystine induces S-phase arrest and apoptosis in human breast adenocarcinoma MCF-7 cells by modulating ERK and Akt phosphorylation. J Agric Food Chem 56: 10574-10581, 2008.

11. Lee EJ, Moon GS, Choi WS, Kim WJ and Moon SK: Naringin-induced p21WAF1-mediated G(1)-phase cell cycle arrest via activation of the Ras/Raf/ERK signaling pathway in vascular smooth muscle cells. Food Chem Toxicol 46: 3800-3807, 2008

12. Lee SJ, Kim HM, Cho YH, Park K, Kim EJ, jung KH, et al: Aqueous extract of Magnolia officinalis mediates proliferative capacity, p21WAF1 expression and TNF-alpha-induced NF-kappaB activity in human urinary bladder cancer 5637 cells; involvement of p38 MAP kinase. Oncol Rep 18: 729-736, 2007.

13. Olazabal I, Munoz J, Ogueta S, Obregon E and Garcia-Ruiz JP: Prolactin (PRL)-PRL receptor system increases cell proliferation involving JNK (c-jun amino terminal kinase) and AP-1 activation: inhibition by glucocorticoids. Mol Endocrinol 14: 564-575, 2000.

14. Schwertfeger KL, Hunter S, Heasley LE, Levresse V, Leon RP DeGregori J and Anderson SM: Prolactin stimulates activation of c-jun N-terminal kinase (JNK). Mol Endocrinol 14: 1592-1602, 2000.

15. Gonzalez JM, Navarro-Puche A, Casar B, Crespo P and Andres V: Fast regulation of AP-1 activity through interaction of lamin A/C, ERK1/2, and c-fos at the nuclear envelope. J Cell Biol 183: 653-666, 2008.

16. Royuela M, Rodriguez-Berriguete G, Fraile B and Paniagua R: TNF-alpha/IL-1/NF-kappaB transduction pathway in human cancer prostate. Histol Histopathol 23: 1279-1290, 2008.

17. Wu W, Chen YH, Ueda E, Tan D, Bartolini P and Walker AM: Different forms of prolactin have opposing effects on the expression of cell cycle regulatory proteins in differentiated mammary epithelial cells. Oncol Res 16: 75-84, 2006. 